\title{
Sedation for an Adult with Mucopolysaccharidosis VI
}

\section{Gloria Merces Leitao Lobo de Araujo Lobo $\mathbf{M}^{1 *}$ and Mourao $\mathrm{J}^{2}$}

${ }^{1}$ Mercês Wolf, Anesthesia Resident, DepartamentofAnesthesia and Intensive Care Portuguese Oncology Institute of Porto Francisco Gentil, EPE, Porto, Portugal ${ }^{2}$ Anesthesiologist, Department of Anesthesia, Hospital Centre S. Joao, EPE, Porto Invited assistantat Faculdade Medicina Dentária of the University of Porto, Voluntary assistant at Faculty of Medicine, University of Porto, Portugal

\begin{abstract}
We describe the sedation of a 22 year old man with mucopolysaccharidosis VI scheduled for a myringotomy procedure. His medical history was significant for difficult airway, severe aortic insufficiency and adenoid hypertrophy. We briefly review the anesthesia literature related to this case.
\end{abstract}

\section{Introduction}

Mucopolysaccharidosis VI or Maroteaux-Lamy syndrome is an inherited, progressive Metabolic Disease, associated with a high degree of anesthetic risk $[1,2]$.

The decreased amount or function of the enzyme $\mathrm{N}$-acetylgalactosamine-4-sulfataseleads to progressive accumulation of glycosaminoglycans in all tissues and organs leading to a multisystemic disorder, clinical manifestations include [3].

- Severe airway obstructions: Obstructive Sleep Apnea, Pneumonia, Hearing Loss

- Skeletal deformities: Spinal Cord or Nerve Root Injury, Joint Abnormalities, Growth Impairment

- Cardiopathy: Valve Disease, Cardiomyopathy, Endocarditis, Electrocardiographic Abnormalities, Systemic Vascular Narrowing and Hypertension

- Ophthalmologic: Blindness and Decreased Visual Acuity, corneal opacification, Retinopathy, Optic Nerve Abnormalities, Ocular Hypertension and Glaucoma [4]

- Central and Peripheral Nervous System-Related Symptoms: is not typically associated with progressive impairment of mental status, but Carpal Tunnel Syndrome, Communicating Hydrocephalus and Compressive Myelopathy are frequent.

Mucopolysaccharidosis VI is associated with a high degree of anesthetic risk [1,2], and airway establishment and maintenance represents the most frequent problem [5-7].

Patients present with severe airway obstructions, cranio-facial abnormalities, short neck, stiffening of the temporomandibular joints, anteriorly positioned larynx, unstable atlantoaxial joint, requiring limited hyperextension of the neck all of which may complicate laryngoscopy and intubation [3].

A survey of airway complications in childens with Mucopolysaccharidosis at the Royal Manchester Children's Hospital (Manchester, United Kingdom) [8] showed an overall incidence of difficult intubation of $25 \%$ of all subgroups and a failed intubation rate of $8 \%$; failed tracheostomy and respiratory arrest have also been reported [6,7].

As the patients grow older, the deposition of glycosaminoglycans in the pharyngeal tissues and tracheal cartilages becomes larger, conditioning intraluminal narrows of the conductive airway, leading to increase difficulties in airway establishment.

Difficult airway should always be anticipated, and a thorough exam of the upper-airway is mandatory, careful observation of chest X-ray, baseline brain and spine MRI, and radiographic views of the cervical spine in flexion and extension may be proven helpful to disclose airway abnormalities. It is also helpful to involve an otolaryngologist in airway evaluation and management [3]

Cardiac system should be fully evaluated before any procedure that requires sedation or anesthesia [4], monitoring should be adapted to the cardiac status.

Spontaneous breathing induction is the corner piece technique in these patients [8] extubation may be as complicated as induction. Pulmonary hypertension and pulmonary edema have been reported in the postoperative period. Always consider referral to a center with anesthesia experience in caring for these patients [3].

\section{Case Report}

A 22 year old man, with a history of Mucopolysaccharidosis VI was scheduled for a myringotomy procedure. His medical history was significant for severe aortic insufficiency, adenoid hypertrophy and chronic otitis media.

He presented with a 2 year history of chronic otitis media and bilateral conductive hearing loss. His past surgical history included a scoliosis correction surgery, bilateral carpal tunnel release and myringotomy. His last anesthetic was 3 years before admission, and a general anesthesia was performed. Orotraqueal intubation was accomplished under fiber-optic visualization and more than 4 attempts were registered. There were no records of complications. For the last four years he has been medicated with galsulfase once a week (Figure 1).

On physical examination the patient was $145 \mathrm{~cm}$ and weighed $60 \mathrm{~kg}$. Airway evaluation: abnormal neck anatomy, short neck with limited mobility, hyromental and thyromentonian distance less than 6 $\mathrm{cm}$, severely limited jaw protrusion, mouth opening inferior to $6 \mathrm{~cm}$, mallampati IV and snoring.

His vital signs were heart rate 50, blood pressure 122/30 $\mathrm{mmHg}$ and

*Corresponding author: Gloria Merces Leitao Lobo de Araujo, Mercês Wolf Anesthesia Resident, Departament of Anesthesia and Intensive Care Portuguese Oncology Institute of Porto Francisco Gentil, EPE, Porto, Portugal, Tel: 00351-9199-281-4; E-mail: merceslobo@gmail.com

Received October 05, 2013; Accepted November 18, 2013; Published November 20, 2013

Citation: Gloria Merces Leitao Lobo de Araujo Lobo M, Mourao J (2013) Sedation for an Adult with Mucopolysaccharidosis VI. J Clin Case Rep 3: 320. doi:10.4172/2165-7920.1000320

Copyright: (c) 2013 Gloria Merces Leitao Lobo de Araujo Lobo M, et al. This is an open-access article distributed under the terms of the Creative Commons Attribution License, which permits unrestricted use, distribution, and reproduction in any medium, provided the original author and source are credited. 


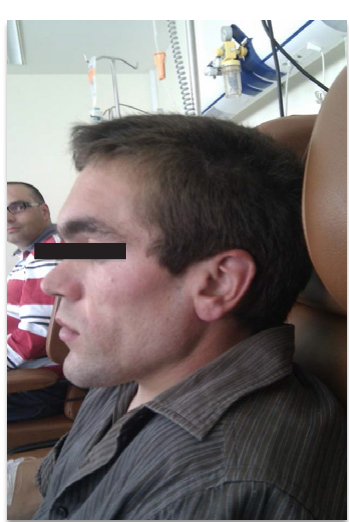

Figure 1: Patient with Mucopolysaccharidosis VI.

respiratory rate 16 . Laboratories and chest $\mathrm{X}$ ray were unremarkable Echocardiogram revealed a severe aortic insufficiency, ejection fraction of 50\% and PSAP $29 \mathrm{mmHg}$. Pulmonary function tests were normal.

Risks and benefits of the surgery were assessed by a multidisciplinary team (otolaryngologist, anesthesiologist and cardiologist) and thoroughly discussed. The patient was informed that his medical condition was of high risk, and informed consent was obtained.

On the day of the surgery at the arrival at the operating room, an $20 \mathrm{G}$ intravenous line was established, ASA Standard II monitoring, nasal canula was placed with oxygen $2 \mathrm{l} / \mathrm{min}$ and a $18 \mathrm{G}$ radial artery was placed under local anesthesia (lidocaine $2 \%$ ). The patient was positioned supine, fentanil 0, $05 \mathrm{mg}$, and propofolbolus of 5-10 mg were administered achieving Moderate Sedation [5]. Patient responded to verbal commands whenever stimulated.

No interventions were required to maintain a patent airway, and spontaneous ventilation was adequate with saturations $98-100 \%$. Cardiovascular function was stable during the procedure, and did not required the use of vasoactive drugs.

\section{Discussion}

From the perioperative assessment a difficult airway was anticipated. Our approach included multiple concerns, as we knew that he had been very difficult to incubate 3 years before, and it would had been probably more difficult to incubate at this time because of the continuous deposition of glycosaminoglycans. Adequate spontaneous ventilation was a primary objective, as face mask ventilation was also likely to be very difficult because of the continuous deposition of glycosaminoglycans, disturbed anatomy and sleep apnea.

We decided to perform a moderate sedation as we expected to be possible to perform the procedure under sedation. If the patient was not able to tolerate the surgical procedure, orotraqueal intubation would be performed under fiber-optic visualization and general anesthesia induced. We also ensured that if we were not able to incubate under fiber optic visualization an experienced otolaryngologist was available to perform emergency tracheostomy.

The airway approach was discussed between pairs (anesthesiologist and otolaryngologist) and with the patient. A clear description of the different methods was made: sedation, awake fiber-optic intubation, and traqueostomy under local anesthesia and the patient were well aware of what could happen in the day of the surgey.

In addition to the airway management we also had to consider the management of the severe aortic insufficiency, we began with the insertion of an arterial line and we chose a low dose fentanyl and propofol for the good recovery characteristics and cardiostability profile.

It is our intention with this case report to raise awareness in the medical community for the anesthesia difficulties and challenges that these patients represent.

\section{References}

1. Linstedt $U$, Maier C, Joehnk H (1994) Threatening spinal cord compression during anesthesia in a child with mucopolysaccharidosis VI. Anesthesiology 80 227-229.

2. Hak Suh S, Okutani R, Nakasuji M, Nakata K (2010) Anesthesia in a patient with mucopolysaccharidosis type VI (Maroteaux-Lamy syndrome). J Anesth 24: 945-948.

3. Giugliani R, Harmatz P, Wraith J (2007) Management Guidelines for Mucopolysaccharidosis VI. Pediatrics 120: 405-418.

4. Fahnehjelm KT, Chen E, Winiarski J (2011) Corneal hysteresis in mucopolysaccharidosis I and VI. Acta Ophthalmol 90: 445-448.

5. Definitions of Sedation (Approved by ASA October 13, 1999).

6. Moores C, Rogers JG, Mckenzie IM, Brown TCK (1996) Anesthesia for children with mucopolysaccharidoses. Anaesth Intensive Care 24: 459-463.

7. Baines D, Keneally J (1983) Anesthetic implications of the mucopolysaccharidoses a fifteen-year experience in a children's hospital. Anaesth Intensive Care 11: 198-202.

8. Walker R, Allen D, Rothera M (1997) A fibreoptic intubation technique for children with mucopolysaccharidoses using the laryngeal mask airway. Paediatr Anaesth 7: 421-426. 